\title{
Related Genes Research Advances of Cotton Male Sterility
}

\author{
Dongmei Liu
}

Shangqiu Normal University,Henan province key laboratory of university in Interaction between plant and microorganism, Shangqiu City, Henan Province, 476000

\author{
Keywords: Related Genes Research, Advances, Cotton Male Sterility
}

\begin{abstract}
The cotton male sterile line is a sterile line formed by the mutation of the nuclear gene that controls the normal fertility of pollen. It is widely used in hybrids because of the advantages of abortion, wide recovery and easy screening of high-yielding hybrids produce. However, there is not much research on the mechanism of cotton male sterility in cotton. In order to better carry out the study of nuclear male sterile materials, the research progress of cotton male sterility mechanism was reviewed from the aspects of morphology, cytology, physiology and biochemistry and molecular basis. The application of nuclear male sterile hybrid cotton and the development direction of cotton male sterility mechanism are put forward.
\end{abstract}

\section{Introduction}

Cotton, as one of the world's most important economic crops, also has obvious heterosis as other crops. China is the first use of cotton genetically modified lines to prepare hybrids and artificial seed production using F2 advantage of cotton producing countries. In the production, the heterosis of cotton is mainly through the two methods of artificial hybridization and the use of cotton male sterile lines. In the breeding of hybrid cotton varieties, the cytoplasmic male sterile lines are very few, the breeding cycle is long and the yield, quality, resistance and other comprehensive traits are not ideal, in the production of large-scale promotion of very few varieties. In contrast, the nuclear male sterile line has a larger application scale, our scholars since the 20th century, since the 1970s, has developed Dong A, Sichuan A, no 62-1A more than 30 kinds of nuclear control by the cotton Male sterile lines or materials. The use of nuclear male sterility, can reduce the labor to reduce labor costs, the use of hybrid generation of disease resistance, high quality, high yield and other advantages in one, so the cotton core male sterile line research has a very big significance, in the Production has a wide range of development prospects. Therefore, many scholars have adopted a variety of research methods from the cell morphology, metabolic components and molecular level on the cotton male sterile line mechanism has been studied, has made some progress.

\section{The Related Research on Cotton Male Sterility}

Cotton is an important economic crop, people's production and life and the national economy plays a decisive role. According to statistics, in 2007 China's cotton acreage area of 5.59 million hectares, accounting for about $15.7 \%$ of the world's cotton planting area, the total output of 7.6 million tons, accounting for about $29 \%$ of the world's total output, consumption of the world's cotton industry, The amount of 935.35 million tons. There is a big gap between supply and demand and conventional cotton production has been far from meeting the needs. Hybrid cotton as a result of substantial increase in yield, the prospects are broad. At present, hybrid cotton seed production there are two main methods, one is to use artificial hybrid method, this method of preparation of free, the preparation of high-yield hybrid probability, but the cost is high, the amount of labor; Male sterile lines produce hybrids, which eliminates the trouble of artificial rosters, but the female is subject to certain restrictions, and still need artificial pollination, while requiring identification and removal of fertile plants in the budding stage, waste of human and land resources. Cotton 
Cytoplasmic Male Sterile Plant Pistils Normal Stamens are completely aborted, which can be improved through the transformation, transformation of some of its bad traits, is the use of hybrid advantages, creating hybrids of the more ideal material.

Cytology Studies. The general morphological characteristics of the CMS CMS are: the sterile lines are small, the stamens are short, the others are shriveled, the stigma length is short, and the size of the ovules before fertilization is small. From a large number of studies, microspore abortion is likely to occur from the sporophytic tissue to the pollen maturation stage. Murthi and Weaver (1974) analyzed the abstinence of Hackney West cotton CMS, suggesting that male malnutrition occurs during sporulation of cells to microspore cell formation. Thombre and Mehetre found a germline cotton cytoplasmic male sterile line, most of its drug room without pollen mother cell development, individual pharmacies despite pollen mother cells, but are disintegrated before meiosis. Liu Jinlan and so on to MA sterile line and "hole A" sterile line as the material, using paraffin section scanning electron microscopy observation technique, observed pollen mother cell callose deposited too much, dissolve slower. The pollen cytoplasm of the mononuclear stage is completely dissolved, but the nucleus remains at different levels, and the nuclear membrane is still clearly visible. Abortion pollen wall development is not complete, the wall thorns protruding small and thin, no germination hole. At the same time, it was observed that the pollen of male sterile strain of hole A was aborted in the whole process of development. The main manifestation of abortion was cytoplasmic hyperplasia and "cytoplasmic wall".

Physiological and Biochemical Research. The current study has found that the expression of male sterile genes has a strict temporal and spatial order. The expression of sterile genes not only involves anatomical abnormalities in anthers, but also reflects changes in their biochemical metabolism. Normal biochemical metabolism is the basis for the material and energy required for normal development of pollen, otherwise the pollen will degrade or die. The production of male sterile lines in cotton is necessarily caused by abnormal changes in certain substances such as enzymes, proteins, degraded substances or metabolic processes. Therefore, people have long tried to compare the differences in the metabolic processes of sterile pollen and fertile pollen. Physiological and biochemical research on the mechanism of male sterility in cotton, and got some preliminary conclusions.

Some of the phenomena of plants must be associated with certain physiological and biochemical metabolites and metabolic enzymes in the abnormal dynamic changes in contact and the production of cytoplasmic male sterility is no exception. Servella and Stojunovic first studied the physiological and biochemical characteristics of cotton CMS, and found that the content of acid amino acid in the sterile anthers of Asian cotton CMS was lower, while the contents of aspartic acid and arginine in leaves were higher. There are few studies on the distribution of starch granules in CMS anthers, the lack of characteristic bands of amylase isoenzymes and the decrease of enzyme activity, and the synthesis of sterile pollen protein in the process of meiosis, and the four amino acids (proline Acid, cysteine, aspartic acid and glutamic acid) abnormalities, causing a metabolic disorder, which is leading to male abortion physiological and biochemical basis. Many scholars have studied the relationship between plant hormones and male sterility. (IAA) content decreased with the decline of fertility, and it was concluded that the male sterility occurred in the IAA library of sterile anthers, and the pollen was aborted.

Molecular Studies. In the study of cotton sterile lines, the current research on gene expression related to pollen development is carried out too little, and the genetic rule is not fully understood. Cotton Clones related to male gametophyte development have not been reported.

With the rapid development of DNA cloning technology and analytical technique and the establishment of organelle separation technology and in vitro translation system, the research on CMS at the molecular level has been greatly promoted. The research on CMS sterile genes is mainly focused on mitochondria, and the mitochondrial genes related to CMS have been identified in many plants. The research on cotton is not deep enough, and the research is focused on searching for sterile lines and the difference between the maintainer and the search for the sterile-related genes. Yu Zhihua and other RAPD techniques were used to detect the polymorphism of cotton cytoplasmic 
male sterile line and maintainer line. Amplification of a characteristic band with OPAH3 primer and good reproducibility showed that the characteristic band was linked to fertility. The results showed that the DNA breeding pool and the DNA sterile pool were established by the combination of NIL and BAS in the cotton breeding group of Nanjing Agricultural University. Through the screening of 524 RAPD random primers, genes related to molecular markers.

Restore gene research. There are three main methods in hybrid cotton seed production, one is to use artificial breeding method, the second is the use of nuclear male sterile line preparation method, the third is the use of cotton cytoplasmic male sterile line. The use of cotton three lines is the best way in comparison. The breeding of cotton restorer lines is the focus of the research on three lines-breeding. Researchers have carried out the study on the restoring genes of four different types of cytoplasmic male sterility of cotton, such as cytoplasmic male sterility D8,Gossypium harknessii,Jin A and 104-7A, respectively, and obtained close linkage with the restorer gene of the marker sites[1].The restorer line AM-R1 from the United States contains two recovery genes, Rf1 and RF2, Rf1 is located between BNL3535 and CIR179 at a distance of $5.3 \mathrm{~cm}$ and RF2 is positioned between STS659 and BNL1045 at a distance of 4.8CM; and the restorer line of Israel Ch-R1 and Is-R1 have a common response gene Rf, located between CIR222 and BNL632, at a distance of 6.7cM[2] .Three RAPD markers and one SSR marker were found by RAPD, AFLP, STS, CAPS and SSR molecular markers techenology, and it was deduced that Rf1 and Rf2 were located on the LGD08 linkage group (named D5 chromosome) of the D subchromosome group [3].Cao found that 13 pairs of SSR primers had obvious polymorphism, and 13 pairs of primers were used to amplify the near-isogenic lines and F2 population, and the Rf1 linkage map was constructed respectively. By comparing the linkage map and analyzing the location of these markers on cotton chromosomes, results also showed that seven markers were on the 19th chromosome (also known as D5 chromosome)among the 13 SSR markers that were linked to Rf1, And these 13 markers linked to the sexual recovery gene Rf1 may be located on the 19th chromosome[4].A large number of CMS recovery gene studies have shown that the gene locus of recovery is likely to occur in a complex form. For example, the recovery gene of kidney beans has two completely different recovery mechanisms, but is located on the same Loci. There were three types of CMS genes, such as BT type and HL type, all located in the same locus. Similarly, nap and pol genes of rapeseed were also mapped to the same locus [5] , The type $P$ and $G$ restorer genes of the rye were located in the same region as the wheat T-type sterile restorer gene.

Study on the mechanism of cytoplasmic male sterility fertility recovery depends on the cloning of Rf gene. Cloning the recovery gene is a challenging task due to the absence of homologous sequence and protein sequence data, common gene cloning strategy is difficult to apply; plant fertility recovery phenotype is the results of the combined effect through the restoration of genes and CMS factors ,So that the analysis of the recovery gene becomes complicated.It has been shown that some of the restorer genes can restore pollen fertility by altering the expression of mitochondrial related genes. The recovery of genes can influence the transcription of mitochondrial CMS gene and then affect the expression of CMS gene.The flanking sequence of the mitochondrial functional gene was cloned and sequenced by using the male sterile line and its maintainer and restorer line of Gossypium harknessii cytoplasmic. Through the bioinformatics analysis, at the downstream of atp4 found a ORF named orf 160, which was unique to the cytoplasm of the Gossypium harknessii[6].Wu[7] cloned the cotton cytoplasmic male sterility fertility recovery gene ZH46-3437, and constructed the overexpression vector with pBI121 vector.Transgenic plants showed semi - sterile traits. Relative fluorescence quantitative data showed that the relative expression of ZH46-3437 decreased, indicating that ZH46-3437 gene may be related to cotton pollen fertility.At present, the study of fertility recovery gene of cotton cytoplasmic male sterility is still in the exploratory stage. The expression of Rf gene leads to the decrease of mitochondrial CMS factor expression, but this process is not clear.Therefore, we can focus in from the following three aspects of in-depth study in the future. First, the further fine positioning of fertility restoration gene for the cloning of fertility recovery related gene loci to provide favorable conditions.Secondly, it is necessary to clarify the Rf protein action model and carry out more detailed biochemical research 
on its function. There is a lack of CMS in Arabidopsis, so the genetics of this model is excluded. However, approximately $20 \mathrm{Rf}$ homologues are identified to provide an opportunity to detect other functions. The sequence data of Arabidopsis thaliana and Brassica plants will play an important role in understanding the evolution of Rf gene clusters.Thirdly, the recovery gene encodes the PPR protein, and the function of the PPR gene should be further studied. In order to analyze the function of a single PPR protein, more mutants need to be sought in traditional studies, but mutations are not easy to screen because many mutants are lethal or have no significant difference in phenotype. However, with the completion of different biological genome sequencing and the development of bioinformatics, it will provide a convenient technical platform for inheritance and function in-depth study of PPR gene .

\section{The Application of Cotton Male Sterility}

Breeding of Cotton Male Sterile Hybrid. China is an early nuclear male sterile line "a dual use" of the use of cotton heterosis in the country, in many nuclear sterile genes, has been put into production and still only use the hole type A (ms14), ms5ms6 and new cultivation Out of the sterile line, other types of nuclear sterility due to or comprehensive traits, or with low compatibility, or pollen abortion is not complete, it is difficult to apply in production. In order to meet the production needs, the transformation of the existing CMS and the combination of advantages of the activities never stopped. Sichuan Province, cotton from the 20th century, 70 years on the basis of the study of self-selected cotton latent sterile line on the basis of inheritance, the use of brother and sister cross, breeding "hole $\mathrm{A}$ " and "473A" nuclear sterile dual- On the basis of 473A sterile line, we entered the anti-disease factor, and after 10 years, the anti-fusarium wilt resistance to Fusarium oxysporum was used as anti-A2, anti-A3, GA, GA18 And so on, on the basis of the selection of a number of production in the promotion of the application of hybrid varieties, such as Sichuan Chuanmian 11, Sichuan Chuan cotton 12, Sichuan Sesame cotton 13, Sichuan Sesame cotton 14, In 1987, the successful cultivation of the male sterile sterile line (MB) was broken down, breaking the limitation of the application of the "three lines" seed production in the nuclear sterile line, and opened up a new way for the application of the nuclear sterile line. Of the hybrid combinations have Chuanyou 1 and so on. Zhu Zhenming and other fertility in 2002 to develop a stable, good adaptability of high quality and disease resistance genic male sterile line too 177A, and the use of the CMS line to cultivate hybrid cotton E cotton 8, Huaying 103 in the production of the application. Hunan Cotton Research Institute through the far - source hybridization method of breeding Xiang cotton CMS line 1 , and the use of Xiang cotton CMS line 1 cultivated Xiangza cotton 9 in the production of use. As a result of the sterile line has a very high yield, anti-wind bell, no nectary and other advantages, many breeding units through hybridization, backcrossing and other methods have been developed to fight pests, cold, drought and infertility Varieties (lines) and cultivate hybrid cotton in the production of applications, such as cotton in the 38, in the cotton 54, Nannong 98-4, 6.

Study on Marked Sterile Lines. The biggest problem with the use of nuclear male sterile lines is that $50 \%$ of the fertile plants need to be removed at the time of seed production, resulting in severe seedling rupture in the field and affecting seed production. Through the identification of traits, it is possible to remove the fertile plants during the seedling stage and the seedling stage. It is an important technical obstacle that must be overcome in the application of the two lines. Therefore, the research on the labeled sterile line is very important at home and abroad. In view of the failure of complete nuclear male sterile (ms2) sterility and partial malformations of leaves, Quisenberry and Kohel conclude that ms2 sterility and leaf malformations are not closely linked as a result of multiple effects. Zhang et al. [60] found a single recessive genic male sterile line 81A (ms16) with a bud-yellow marker trait, and that bud-yellow traits and male sterility were all controlled by a pair of recessive genes Showing a close chain or complete chain or a multi-effect. Pan Jiaju and other on the application of onion cotton bud yellow gene in the hybrid cotton feasibility study, that can be used to overcome the nuclear male sterile line until the flowering period to identify the defects of sterile and fertile plants, improve seed production and effectiveness. Lu Shuping et al reported the establishment of a nuclear male sterile line closely linked to the anti-kanamycin marker trait. 


\section{Current Problems and Perspectives}

Although some studies have been done on the mechanism of cotton and male sterility, some progress has been made in cell morphology, physiology, biochemistry and molecular biology. However, there are still many problems to be solved, and further research is needed. Cotton sterile pollen abortion will last for a long time, from the early emergence of sporulation cells to dual-core pollen grains have microspore abortion, and abortion of pollen will have some of the same abnormal performance, but still It is not certain which cell morphology is a typical feature of pollen abortion.

Although it can be determined that the abnormalities of physiological and biochemical factors such as starch, protein synthesis and amino acid composition lead to the occurrence of male sterility, but which is the dominant factor, and how to play a role, the relationship between mineral element content and nuclear male sterility, these are to be further studied. In the study of cotton sterile lines, the current research on gene expression related to pollen development is carried out too little, and the genetic rule is not fully understood. How nuclear genes affect pollen development and the mechanism of action of sterile genes are worthy of further study in our future work.

\section{Conclusion}

Hybridization of cotton male sterile lines is one of the directions of hybrid cotton application. The promotion of male sterile hybrids can solve the phenomenon of F2 filling F1 and conventional hybridization in the current intergeneric hybrids. Therefore, the application of nuclear male sterile hybrids is promising, and the key technology is to make breakthroughs in the research and creation of marker sterile lines, to screen out the combination of high advantages and to explore and improve the hybrid production technology.

\section{Acknowledgements}

Fund Project: National Natural Science Foundation of China: No.: 31571997; Henan Provincial Higher Education Key Scientific Research Project Funding Plan: No.: 3157199716A210036

\section{References}

[1] Feng Xue-mei, Liu Feng, Liu Yu-dong, Yan Zu-jun, Han Xiu-lan, Shen fu-fu.Study on New Type of Male Sterile Line 21A in Upland Cotton [J]. Journal of Plant Genetic Resources 2010 (04)

[2] Zou Pei-ping, Wang Qing-ya, Tang Can-ming.Study on Anther Development of Male Latent Male Sterile Line ms5ms6 in Upland Cotton [J]. Acta Chimica Sinica, 2009 (04)

[3] Zhou Zhonghua, Chen Jinxiang, Liu Aiyu, Li Ruilian, Liu Haier, Zhu Quyuan.Studies on Morphological and Cytological Characteristics of Special Cotton S-1 in Cotton Thermo-sensitive Male Sterile Line [J]. Acta Chimica Sinica, 2008 (06)

[4] Tang Wen, Zhang Xiangqiong. Cotton "hole A" type male sterility morphology and mechanism of the status quo[J]. Anhui Agricultural Sciences. 2008 (04)

[5] Gao Junping, Han Guangjin, Gong Yongchao, Wang Furong. Advances in molecular biology of cotton male sterility[J]. Modern Agricultural Science and Technology. 2008 (02)

[6] Liu Zhi, Liu Shaoqing, Yu Xiaonan, Chen Jinxiang. The Cytological Basis of the S-1 Male Sterility and the Response of Fertile Pollen to Temperature[J]. Journal of Hunan Agricultural University (Natural Science Edition) 